Endocrinol. Japon. 1987, 34 (3), 415-422

\title{
Postprandial Changes in Plasma Ketone Body and Carnitine Levels in Normal and Non-Insulin-Dependent Diabetic Subjects
}

\author{
YUKIChI OKUDA, KoICHI KAWAI, YASUKo MURAYAMA \\ AND KAMEJIRO YAMASHITA \\ Department of Internal Medicine, Institute of Clinical Medicine \\ University of Tsukuba
}

\begin{abstract}
We have examined changes in plasma ketone bodies and their related metabolites after the ingestion of a mixed meal in normal and NIDDM (non-insulindependent diabetes mellitus) using a highly sensitive colorimetric method. In normal subjects, fasting plasma acetoacetic acid (AcAc) and 3- $\beta$-hydroxybutyric acid (3-OHB) concentrations were $40.2 \pm 2.9$ and $21.3 \pm 4.0 \mu \mathrm{M}$, respectively. The total carnitine level in fasting plasma was $48.2 \pm 3.2 \mu \mathrm{M}$ and acyl/free was $0.34 \pm 0.12$. These values did not change significantly after the meal. In diabetic subjects, fasting AcAc and 3-OHB levels were 57.9 \pm 3.5 and $97.9 \pm 14.7$ $\mu \mathrm{M}$, respectively and these values especially $3-\mathrm{OHB}$, decreased almost to the normal level by $4 \mathrm{~h}$ after the meal. The total carnitine level in the fasting plasma of diabetics was $48.7 \pm 2.8 \mu \mathrm{M}$ and acyl/free was $0.58 \pm 0.09$. Such characteristics were observed when their plasma glucose had been completely normal for more than 10 days. In patients treated with sulfonylurea, the fasting ketone body level, especially 3-OHB, was significantly lower than that of diabetics treated with insulin or diet alone, whereas the fasting plasma glucose level and its postprandial increase were higher than those of the others. These results demonstrate that measurement of the plasma ketone body (especially 3-OHB) level is a sensitive tool for monitoring the metabolic status of NIDDM.
\end{abstract}

Fasting plasma glucose and glycosylated hemoglobin have been used as markers of metabolic control in diabetics. However, diabetes mellitus is also characterized by abnormalities in lipid and protein metabolism. Ketosis is an important indicator to

\footnotetext{
Received October 27, 1986

Address for correspondence :

Koichi Kawai, M. D. \& Ph. D.

Institute of Clinical Medicine,

University of Tsukuba,

Sakura-mura, Niihari-gun, Ibaraki-ken 305, Japan
}

differentiate between insulin-dependent and non-insulin-dependent diabetes (NIDDM) and determination of plasma ketone body is useful in detecting insufficient insulin therapy and in monitoring the diabetic control. A previous method, the nitroprusside reaction, used in measuring the ketone body concentration, only detects acetoacetic acid (AcAc) and acetone but not 3- $\beta$-hydroxybutyric acid (3-OHB). Accordingly $3-\mathrm{OHB}$ has been assayed byt he enzymatic method (Williamson and Mellanby, 1974). Using this method, it has been 
reported that the plasma ketone body concentration is influenced by fasting (Foster, 1984), exercise (Sato et al., 1983) stress (Kojima et al., 1982), and food intake (Foster, 1984). However, there have been no consistent results concerning the plasma ketone body level and its related metabolites such as free fatty acids (FFA) and carnitine of NIDDM.

The present study was therefore aimed at clarifying the changes in plasma levels of ketone bodies, FFA and carnitine after the ingestion of a mixed meal in normal and NIDDM using a highly sensitive and simplified colorimetric method for differential determination of plasma ketone bodies developed by the modification of Salway's method (Harano et al., 1983 ; Salway, 1969).

\section{Materials and Methods}

\section{Subject}

Ten normal volunteers and 24 NIDDM who were admitted to the university hospital participated in this study. Diabetic subjects were classified into three groups according to the therapy required to control their blood glucose; those on diet therapy alone (D-group), sulfonylurea therapy (S-group), and intermediateinsulin therapy (I-group). The patients, whose fasting plasma glucose (FPG) and plasma glucose $2 \mathrm{hr}$ after the meal were controlled to less than $110 \mathrm{mg} / \mathrm{dl}$ and $140 \mathrm{mg} / \mathrm{dl}$ respectively for more than 10 days during admission, were reexamined and grouped as a well-controlled group. A wellcontrolled group consisted of 9 patients-three from each group. The clinical characteristics of these groups are listed in Table 1.

\section{Protocol}

After an overnight fast, a $400 \mathrm{kcal}$ mixed meal consisting of $51 \mathrm{~g}$ carbohydrate, $22 \mathrm{~g}$ protein and $12 \mathrm{~g}$ fat was given to all subjects. An ordinary sulfonylurea or insulin regimen was employed on the test day. $4 \mathrm{ml}$ samples of blood were drawn into heparinized tubes before, $2 \mathrm{~h}$, and $4 \mathrm{~h}$ after the meal and the plasma was stored at $-20^{\circ} \mathrm{C}$ until assayed. Ketone bodies and FFA were assayed within 1 week.

\section{Analytical Method}

Plasma AcAc and 3-OHB concentrations were determined by the method of Harano et al. (1983) using a commercial kit (Ketone test, Sanwa Kagaku Co., Japan). The plasma glucose concentration was measured by a glucose oxidase method (glucose analyzer 2, Beckman) and the plasma immunoreactive insulin (IRI) concentration was determined by a radioimmunoassay (RIA) according to the method of Herbert et al. (1965). The plasma IRI concentration of insulin-treated subjects was determined after the plasma was treated with polyethylene glycol (Kuzuya et al., 1977). Plasma free and acylcarnitine concentrations were measured by a radioenzymatic method (McGarry and Foster, 1976). Plasma FFA was assayed with a commercial kit (NEFA C test, Wako Junyaku Co., Japan).

\section{Statistical Method}

Data were analysed by analysis of variance followed by group comparisons, Student's $t$-test for paired (comparison within groups) or nonpaired data (comparison between groups), when a significant $F$ value was obtained. $p<0.05$ was considered to be significant. All data in this paper are expressed as the mean \pm SEM.

Table 1.. Characteristics of normal and diabetic subjects

\begin{tabular}{lrccc}
\hline & N & ag (age) & $\begin{array}{c}\% \text { ideal } \\
\text { body Weight }\end{array}$ & $\begin{array}{c}\text { fasting plasma glucose } \\
\text { (mg/dl) }\end{array}$ \\
\hline Normal & 10 & $44.1 \pm 5.1$ & $106 \pm 4.0$ & $94 \pm 2$ \\
Diabetics & 24 & $55.0 \pm 2.6$ & $116 \pm 4.1$ & $148 \pm 10$ \\
Diet & 8 & $53.3 \pm 5.5$ & $118 \pm 10.1$ & $160 \pm 21$ \\
Sulfonylurea & 8 & $58.1 \pm 4.1$ & $114 \pm 9.2$ & $185 \pm 28$ \\
Insulin & 8 & $53.7 \pm 4.1$ & $102 \pm 5.2$ & $139 \pm 17$ \\
Well-controlled diabetics & 9 & $54.2 \pm 4.0$ & $107 \pm 2.0$ & $107 \pm 2$ \\
\hline
\end{tabular}




\section{Results}

Changes in plasma glucose, FFA and insulin levels after the ingestion of a mixed meal (Fig. 1)

In normal subjects, plasma glucose did not change significantly after the mixed meal. The mean fasting plasma glucose levels of the three diabetic groups were not significantly different from each other (Table 1 ), and $4 \mathrm{~h}$ after the meal they returned to near the basal level in the D- and I-group but remained elevated in the S-group.

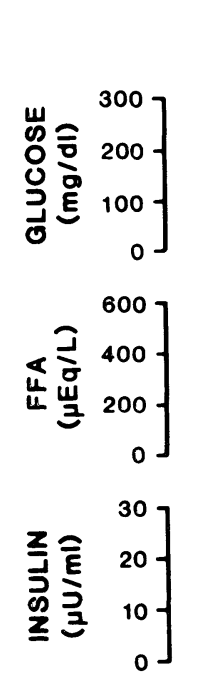

NIDDM

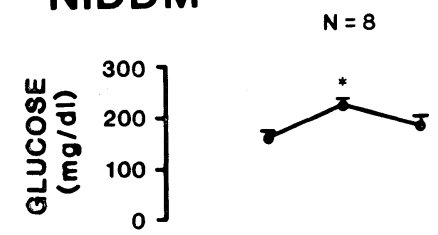

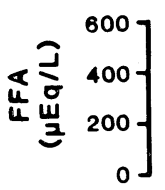
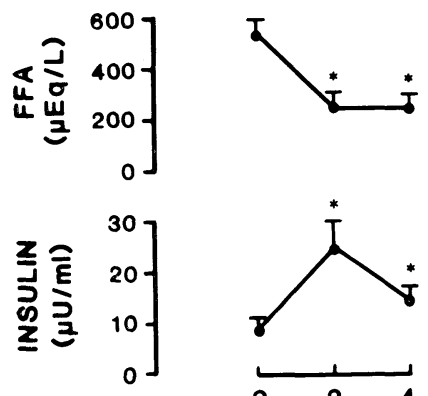

NORMAL

$N=10$
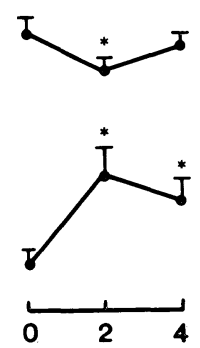

SULFONYLUREA
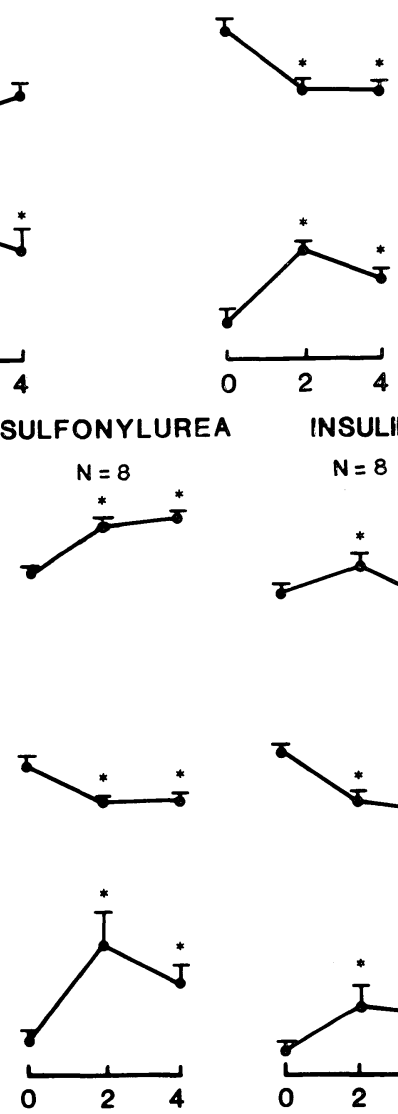

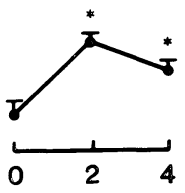

INSULIN

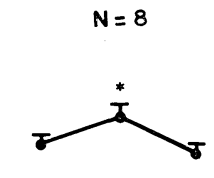

NIDDM
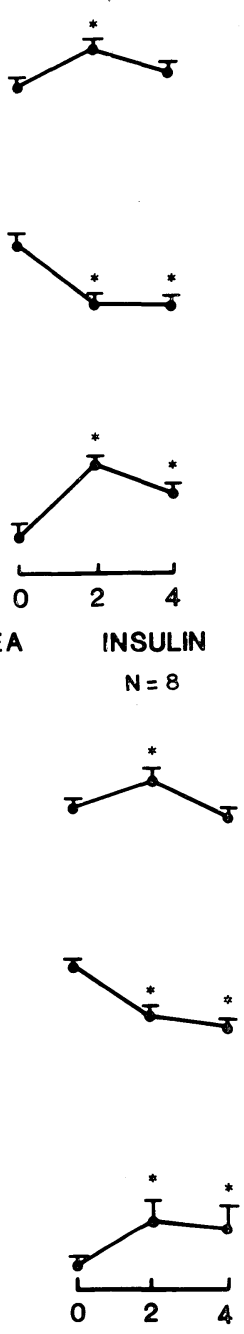

Fig. 1. Change in plasma glucose, free fatty acids and insulin levels in normal and diabetic subjects after the ingestion of a mixed meal. Diabetics refer to the mean of the three groups immediately below. mean \pm SEM. $* \mathrm{p}<0.05$ vs. each basal level.

$$
T, M E(\mathrm{hr})
$$


The plasma FFA of normal subjects decreased significantly $2 \mathrm{~h}$ after the meal $(p<0.05)$ and returned to the fasting level $4 \mathrm{~h}$ after the meal. The fasting plasma FFA level of diabetic patients was significantly higher than that of normal subjects $(439 \pm 34$ vs. $296 \pm 34 \mu \mathrm{Eq} / 1, \mathrm{p}<0.01)$. Two or $4 \mathrm{~h}$ after the meal, the level fell to near that of norml subjects in all groups.

Plasma IRI in both normal and diabetic subjects increased after the meal. The degree of the increase in I-group was smaller than those in the D- or S-group $\mathrm{p}<0.05)$.
Changes in Plasma ketone body level after the ingestion of a mixed meal (Fig. 2)

In normal subjects, fasting plasma AcAc and $3-\mathrm{OHB}$ concentrations were $40.2 \pm 2.4$ and $18.3 \pm 4.3 \mu \mathrm{M}$, respectively. These values did not change significantly during the test period, although the 3-OHB level at $2 \mathrm{~h}$ after the meal tended to fall. In diabetic subjects, fasting plasma AcAc and 3-OHB levels were significantly higher than those of normal subjects: $57.9 \pm 3.5 \mathrm{vs}$. $40.2 \pm 2.7 \mu \mathrm{M}, \mathrm{p}<0.05$, and $97.9 \pm 14.7 \mathrm{vs}$. $18.3 \pm 3.9 \mu \mathrm{M}, \mathrm{p}<0.01$, respectively. The level of the I-group was the highest and that of the S-group was the lowest; total ketone body and 3-OHB levels of the

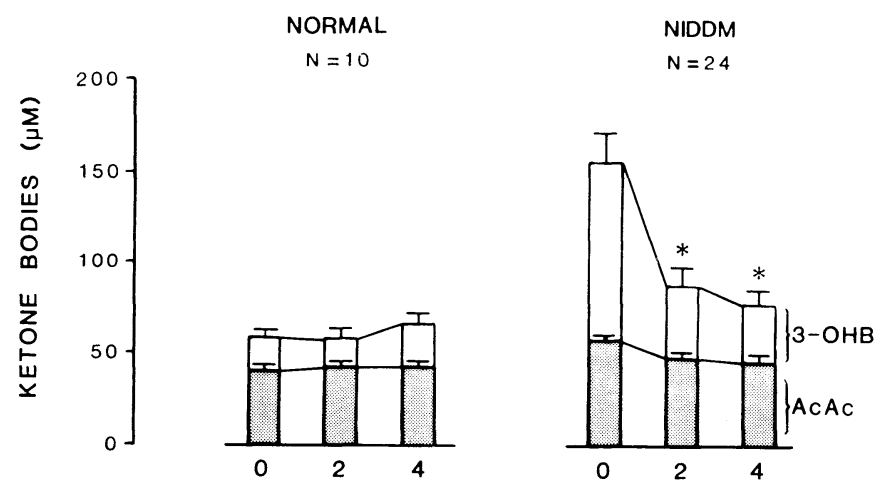

NIDDM

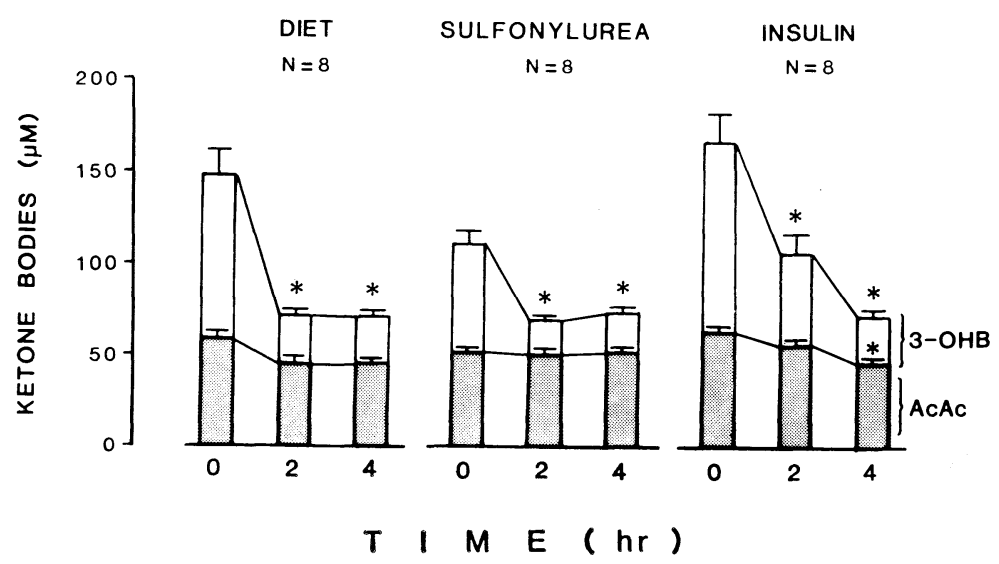

Fig. 2. Changes in plasma ketone body level in normal and diabetc subjects after the ingestion of a mixed meal. Diabetics refer to the mean of the three groups immediately below. mean \pm SEM, Open areas and solid areas represent 3$\mathrm{OHB}$ and AcAc, respectively. ${ }^{*} \mathrm{p}<0.05$ vs. each basal level. 


$\begin{array}{cc}\text { NORMAL } & \text { NIDDM } \\ N=10 & N=24\end{array}$

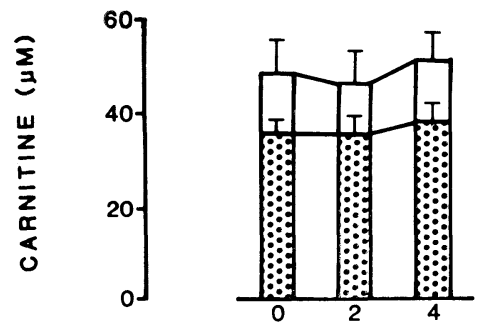

NIDDM
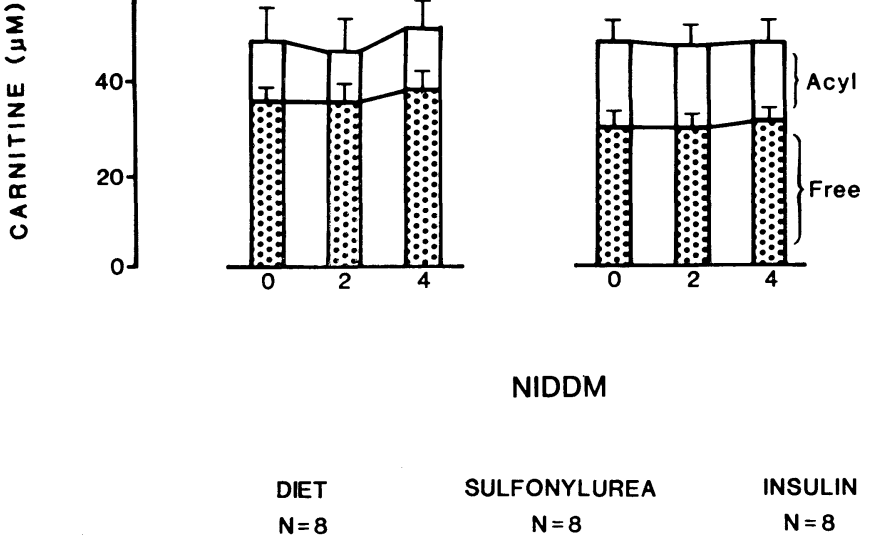

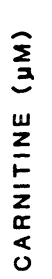
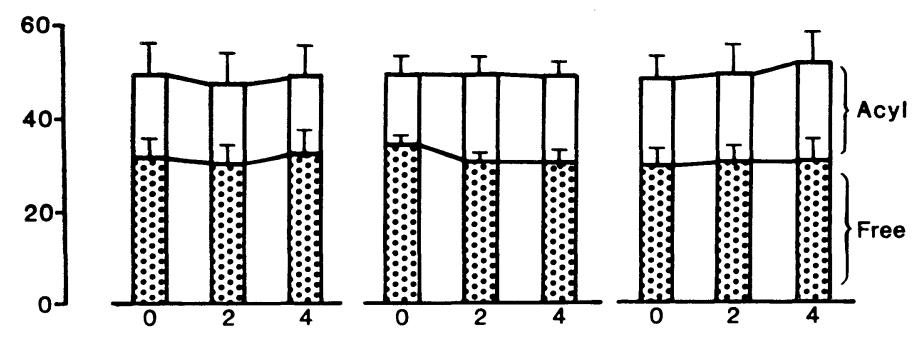

Fig. 3. Changes in plasma carnitine level of normal and diabetic subjects after the ingestion of a mixed meal. mean \pm SEM, Open areas and hatched areas represent acylcarnitine and free carnitine, respectively.

TIME (hr)

S-group were significantly lower than those of the other groups $(p<0.05)$. After the ingestion of the meal, the ketone body level, especially 3-OHB, significantly decreased in all groups and almost reached that of normal subjects $4 \mathrm{~h}$ after the meal.

Changes in plasma carnitine level after the ingestion of a mixed meal (Fig. 3)

The fasting plasma total carnitine level was $48.2 \pm 5.6 \mu \mathrm{M}$ in normal subjects and $47.0 \pm 3.2 \mu \mathrm{M}$ in diabetic subjects. The acylcarnitine/free carnitine ratio was $0.34 \pm$ 0.12 in normal subjects and $0.57 \pm 0.19$ in diabetic subjects $(\mathrm{p}<0.05)$.

The total carnitine level and the acyl/free ratio did not change significantly after the meal in all groups except for the acyl/free in S-group. The fasting acylcarnitine level of the S-group $(14.5 \pm 2.5 \mu \mathrm{M})$ was significantly lower than that of the I-group (19.3 $\pm 2.0 \mu \mathrm{M}, \mathrm{p}<0.05)$, but reached the level of the other groups after the meal.

Changes in plasma ketone body level in wellcontrolled diabetics after the ingestion of $a$ mixed meal (Fig. 4)

The same study was performed in diaabetic subjects whose plasma glucose was completely normalized (FPG $<110 \mathrm{mg} / \mathrm{dl}$, $2 \mathrm{~h}<140 \mathrm{mg} / \mathrm{ml}$ ) for more than 10 days during admission. Their plasma FFA level was lower than that of a fairly controlled state (Fig. 1) and close to that of normal 


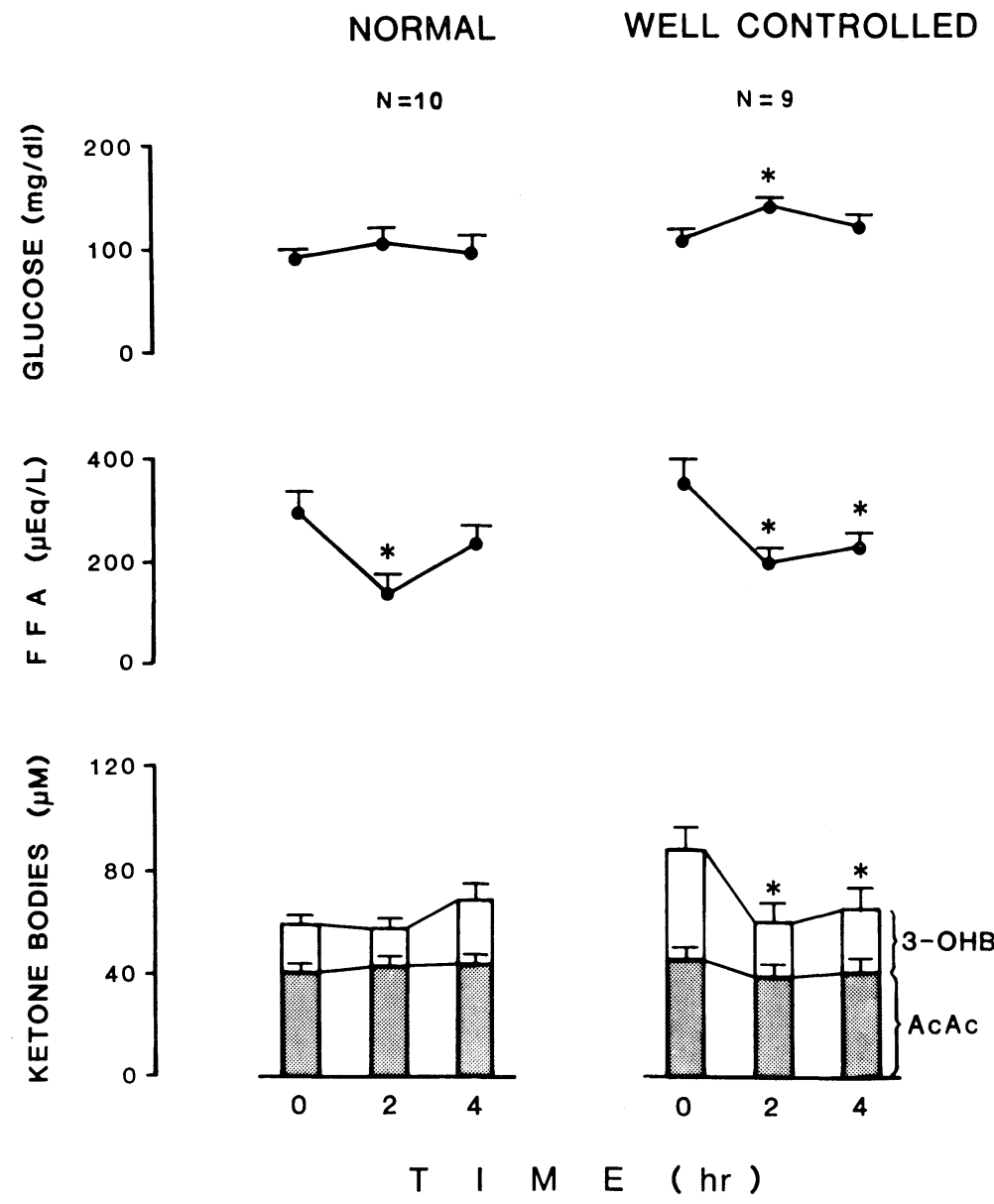

Fig. 4. Comparison of plasma glucose, free fatty acids and ketone body levels after the ingestion of a mixed meal between normal and well controlled diabetics. mean \pm SEM, Open areas and solid areas represent 3-OHB and AcAc, respectively. ${ }_{1}^{*} \mathrm{p}<0.05, \quad$ significatly different from each basal level subjects. The fasting plasma total ketone body was $88.7 \pm 8.3 \mu \mathrm{M}$, which was lower than that for a fairly controlled state $(\mathrm{p}<$ 0.05 , Fig. 2) but significantly higher than that of normal subjects. After the meal, the value, especially that of 3-OHB, quickly decreased almost to the normal level.

\section{Discussion}

The present study demonstrates that plasma ketone body level of NIDDM, especially that of 3-OHB, falls significantly by $4 \mathrm{hr}$ after the ingestion of a mixed meal in parallel with the decrease in plasma FFA. A similar postprandial decrease in the plasma ketone body level was reported by Harano et al. (1984). On the other hand, no significant changes in the plasma ketone body level were observed in normal subjects although the plasma FFA level fell significantly $2 \mathrm{hr}$ after the meal. The ketone body production was essentially regulated by insulin and glucagon through the hepatic malonyl CoA level and the supply of substrate, FFA (McGarry and Foster, 1979). Fig. 1 demonstrates the significant rise in the plasma insulin level after the meal. Although the plasma glucagon 
level was not measured in this study, we observed a significant rise in it for $60 \mathrm{~min}$ after the ingestion of a mixed meal (Kawai et al., Submitted for publication). Therefore, the increased insulin secretion should contribute to the postprandial drop in the plasma ketone body level in NIDDM.

Thus, the relative insulin deficiency in diabetic patients was detectable by either the plasma FFA or the ketone bodies, especially 3-OHB. However, the change in 3-OHB was more sensitive than that in FFA, as shown in Fig. 4. The fasting FFA level of well-controlled diabetics was almost the same as that of normal subjects, whereas the fasting 3-OHB of the former was approximately double that of the latter and its postprandial change was absolutely different. Scheen et al. (1984) demonstrated that the interruption of continuous subcutaneous insulin infusion for $2 \mathrm{~h}$ caused a rise in the plasma 3-OHB level more rapidly than that in the plasma glucose level.

The fasting plasma glucose level of diabetics treated with sulfonylurea was the highest among the three groups, while the ketone body levels, especially 3-OHB, were lower than those of diabetics treated with diet alone or insulin. Harano et al. (1984) also reported that the plasma total ketone body level of diabetics treated with sulfonylurea was the lowest among the fairly controlled NIDDM. These results may be ascribed to an extrapancreatic effect of sulfonylurea and/or a higher insulin concentration in the portal vein under sulfonylurea treatment than that under insulin treatment. Experimentally, Sakamoto et al. (1973) demonstrated that tolbutamide suppressed ketogenesis in normal rat livers and alloxan-induced-diabetic rat livers to onefifth and half of the control, respectively. Boshell et al. (1960) also reported the inhibition of ketogenesis in rat hepatocytes by tolbutamide and they speculated that the inhibitory mechanism may be ascribed to the activation of acetyl-CoA or acetoacetyl
CoA.

Carnitine plays an important role in ketone body production, because it is essential in transporting long-chain fatty acids across the inner mitochondrial membrane (Bremer et al., 1977). The increase in oxidation of fatty acids correlates with the long-chain acylcarnitine concentration in the liver and the plasma carnitine level correlates with the amount of acylcarnitine in the liver (Brass and Hoppel, 1978). Analysing the relationship between plasma carnitines and plasma ketones, Genuth et al. (1979) found a significant correlation ( $\mathrm{r}=$ $0.70, \mathrm{p}<0.01)$ between long-chain acylcarnitine and 3-OHB in insulin-dependent diabetics. However, in this study we have not been able to demonstrate any difference between the plasma acylcarnitine levels in normal subjects and NIDDM.

These results suggest that the changes in the plasma ketone body level (especially 3-OHB) are different from those in the plasma glucose level in various clinical conditions and that the fasting plasma ketone body level and its postprandial changes more sensitively reflect the metabolic abnormality in diabetics than the plasma glucose level does.

\section{References}

Boshell, B. R., G. R. Zahnd and A. E. Renold (1960). An effect of tolbutamide on ketogenesis in vivo and in vitro. Metabolism 9, 21-29.

Brass, E. P. and C. L. Hoppel (1978). Carnitine metabolism in the fasting rat. J. Biol. Chem. 253, 2688-2693.

Bremer, (1977). Carnitine and its role in fatty acid metabolism. Trends Biochem. Sci. 2, 207 $-209$.

Foster, D. W. (1984). From glycogen to ketones and back. Diabetes 33, 1188-1199.

Genuth, S. M. and C. L. Hoppel (1979). Plasma and urine carnitine in diabetic ketosis. Diabetes 28, 1083-1087.

Harano, Y., Kosugi, T. Hyosu, S. Uno, Y. Ichikawa, and Y. Shigeta (1983). Sensitive and 
simplified method for the differential determination of serum levels of ketone bodies. Clin. Chem. Acta 123, 327-336.

Harano, Y., K. Kosugi, T. Hyosu, M. Suzuki, H. Hidaka, A. Kashiwagi, S. Uno and Y. Shigeta (1984). Ketone bodies as markers for Type 1 (insulin-dependent) diabetes and their value in the monitoring of diabetic control. Diabetologia 24, 343-348.

Herbert, V., K-S. Lau, C. W. Gottlieb and S. J. Bleicher (1965). Coated charcoal immunoassy of insulin. J. Clin. Endocrinol. Metab. 25, 1375-1384.

Kawai, K., Y. Murayama, Y. Okuda and K. Yamashita. Postprandial glucose, insulin and glucagon responses to meals with different nutrient compositions in non-insulin-dependent diabetes mellitus. (submitted to Endocrinol Japon)

Kojima, H. (1982). Biochemical changes after surgical 'stress in rabbits. Jpn. J. Surg. 12, 52-60.

Kuzuya, H., P. M. Blix, D. L. Horwitz, D. F. Steiner and A. H. Rubenstein (1977). Determination of free and total insulin and Cpeptide in insulin-treated diabetes. Diabetes 26, 22-29.

McGarry, J. D. and D. W. Foster (1976). An improved and simplified radioisotopic assay for the determination of free and esterified carnitine. J. Lip. Res. 17, 277-281.
McGarry J. D. and D. W. Foster (1979). In support of the roles of malonyl CoA and carnitine acyltransferase $I$ in the regulation of hepatic fatty acid oxidation and ketogenesis. J. Biol. Chem. 254, 8163-8168.

Sakamoto, N., N. Hotta, M. Yoshida, Y. Sato, M. Nakajima, H. Kakuta, A. Iguchi and R. Nomura (1973). Studies on diabetic ketosis (2); Effect of tolbutamide in gluconeogenesis and ketosis in the isolated perfused rat livers. J. Japan Diab. Soc. 16, 275-280.

Salway, J. G. (1969). The simultaneous dertermination of acetoacetate and glucose in capillary blood. Clin. Chim. Acta 25, 109-116.

Sato, Y., N. Sakamoto, L, Hagenfeldt, and J. Wahren (1983). Studies on exercise treatment for diabetes mellitus $(\mathrm{V})$; ketone body metabolism before, during and after exercise. $J$. Japan. Diab. Soc. 26, 127-134.

Scheen, A., M. Castillo, B. Jandrain, G. Krzentowski, P. Henrivaux, A. S. Luycky and P. H. Lefèbvre (1984). Metabolic alternations after a two-hour nocturnal interruption of a continuous subcutaneous insulin infusion. Diabetes Care 7, 338-342.

Williamson, D. H. and J. Mellanby (1974). D(-)-3-hydroxybutyrate and acetoacetate. In: Method of Enzymatic Analysis (H. U. Bergmeyer eds), Academic Press New York. pp. 1836-1843. 